\title{
Von Hockeroda nach New Orleans ...
}

\author{
Andreas Klee $\cdot$ Wladimir Sgibnev
}

(C) Springer-Verlag Berlin Heidelberg 2014

... reichen in diesem Heft von Raumforschung und Raumordnung die Wissenschaftlichen Beiträge in räumlicher Hinsicht. Sie decken nicht nur eine Bandbreite an Untersuchungsräumen $\mathrm{ab}$, sondern stellen eine Reihe von aktuellen raumbezogenen Problemen und methodischen Perspektiven vor. Hockeroda, eine kleine Gemeinde im ländlichen Thüringen, sicherlich nur den wenigsten unserer Leserinnen und Leser bekannt, steht hier für eine Untersuchung des Mobilitätsverhaltens älterer Menschen im ländlichen Raum. Seit der Sensibilisierung der Raumforschung für den demographischen Wandel und seine Herausforderungen befasst sich die geographische Mobilitätsforschung verstärkt mit der Mobilität älterer Menschen, so auch Matthias Wilde in seinem Beitrag „Ach, da fahr ich ganz spontan. Mobilität im Alltag älterer Menschen auf dem Land“. Wilde fasst Mobilität als soziales Problem auf und fragt, wie Mobilität und Lebenswirklichkeit miteinander verflochten sind. Er nähert sich der Alltagsmobilität älterer Menschen am Beispiel der Gemeinde Hockeroda aus einer akteurszentrierten Perspektive und zeigt, wie ältere Menschen auf dem Land ihre Mobilität sehen und in ihren Alltag einbetten. So sind es vorrangig Ereignisse der Zusammenkunft, die das Leben älterer Menschen auf dem Land prägen und die der Mobi-

Dr. A. Klee $(\bowtie)$

Akademie für Raumforschung und Landesplanung - Leibniz-

Forum für Raumwissenschaften,

Hohenzollernstraße 11,

30161 Hannover, Deutschland

E-Mail: klee@arl-net.de

Dr. W. Sgibnev

Leibniz-Institut für Länderkunde,

Schongauerstraße 9,

04328 Leipzig, Deutschland

E-Mail: w_sgibnev@iffl-leipzig.de lität eine spezifische, bislang oft wenig beachtete Funktion verleihen.

Jan Glatter, Katharina Hackenberg und Manuel Wolff wenden sich einer anderen Bevölkerungsgruppe, den Studierenden, zu, insbesondere in ihrer Funktion als Akteure auf lokalen und regionalen Wohnungsmärkten. Auch dies stellt eine Thematik dar, die in den vergangenen Jahren nur an manchen Hochschulstandorten fachliche Aufmerksamkeit hervorrief. Jedoch gewinnen angesichts steigender Studierendenzahlen und zunehmender Wohnungsknappheit Diskussionen über das Segment des studentischen Wohnens in Politik, Medien, Wohnungswirtschaft und Wissenschaft erneut an Aufmerksamkeit. Der Beitrag ,Zimmer frei? Die Wiederentdeckung der Relevanz des studentischen Wohnens für lokale Wohnungsmärkte" von Glatter, Hackenberg und Wolff arbeitet zunächst die Faktoren der steigenden Wohnungsnachfrage Studierender an deutschen Hochschulen heraus und stellt Untersuchungen zum studentischen Wohnen im Hinblick auf ihre wohnungsmarktpolitische Bedeutung vor. Schließlich charakterisieren die Autoren die Entwicklung und Struktur der Angebots- und Nachfrageseite und skizzieren die Folgen der Marktbedingungen auf Mieter- und Vermietermärkten für Studierende.

Olaf Schnur und Henning Günter berichten über „Collaborative Consumption, Sozialkapital und Quartier. Eine Annäherung" und wenden sich mit Collaborative Consumption einem Modell des alternativen Wirtschaftens zu, das statt des Besitzes den geteilten Zugang zu sowohl immateriellen wie materiellen Ressourcen betont und hierbei Gebrauch von webbasierten Interaktionsplattformen macht. Dieses neue Modell, das vielerorts, vor allem in Großstädten, zu beobachten ist, ist einerseits in globale Kommunikationsströme eingebunden, andererseits bedarf es lokaler Gegebenheiten und Gelegenheiten. Damit bewegt sich der Beitrag im Spannungsfeld von Globalität und Lokalität und 
es werden Zusammenhänge dieses Modells mit Konzepten des Quartiers und der Quartiersentwicklung herausgearbeitet. Schnur und Günter entwickeln hierfür eine Typologie verschiedener Collaborative-Consumption-Arten, die sich konzeptionell am Sozialkapital und dessen Verwertbarkeit im Quartierskontext orientiert. Ein eher ernüchterndes Ergebnis daraus ist, dass viele der Angebote nicht notwendigerweise zu einer Akkumulation von lokalem Sozialkapital im Quartier führen müssen, was vielerorts jedoch angenommen wird.

Der vor über zwei Jahrzehnten eingeleitete Paradigmenwechsel der Förderpolitik für ländliche Regionen hin zu einer kooperativen, akteursorientierten Regionalentwicklung ist der Anlass für Christian Diller, Guido Nischwitz und Benedict Kreutz zu überprüfen, wie wirksam diese Förderansätze für die Regionalentwicklung sind. Denn bislang gibt es kaum Untersuchungen, die den Zusammenhang des Einsatzes von Programmen der kooperativen Regionalentwicklung (,Regional Governance“) mit gängigen regionalen Entwicklungsindikatoren thematisieren. Die Autoren zeigen in ihrem Beitrag „Förderung von Regionalen Netzwerken: Messbare Effekte für die Regionalentwicklung?“, dass nachhaltige Wirkungen für die Regionalentwicklung praktisch nicht messbar sind. Die vorgenommenen statistischen Auswertungen liefern keine Hinweise darauf, dass sich Regionen, in denen verstärkt Regionalinitiativen gefördert wurden, in der Einwohnerzahl und Wirtschaftskraft besser entwickelten als jene, in denen dies nicht geschah. Dieser bedenkenswerte Befund stellt viele bisher unhinterfragte Annahmen auf den Kopf und sollte Anstöße für die
Weiterentwicklung dieser Förderinstrumente ländlicher Entwicklung geben.

Mark Kammerbauer wendet sich im letzten Wissenschaftlichen Beitrag der Stadt New Orleans in den Vereinigten Staaten zu und untersucht, welche Herausforderungen die Stadt beim Wiederaufbau nach den durch den Hurrikan Katrina 2005 ausgelösten Überschwemmungen zu meistern hatte. Kammerbauer macht in seinem Beitrag „Asymmetrischer Wiederaufbau in Städten nach Katastrophen. Das Lower Ninth Ward in New Orleans nach Orkan Katrina" deutlich, dass der Umgang mit den Folgen des Schadensereignisses ein sehr komplexes Handlungsfeld darstellt, das den städtischen Raum, intervenierende staatliche Institutionen und nicht zuletzt betroffene Bürger umfasst. Im Quartier Lower Ninth Ward in New Orleans sollte eigentlich ein auf Hauseigentümer ausgerichtetes Wiederaufbauprogramm den Bürgern die Rückkehr erleichtern. Aber es zeigte sich, dass die entsprechenden Pläne und Programme zu unterschiedlichen Geschwindigkeiten und Intensitäten des Wiederaufbaus führten. Kammerbauer begründet dies damit, dass die Pläne und Programme nicht adäquat auf städtebauliche und soziale Rahmenbedingungen reagierten und nicht auf die Verwundbarkeit der betroffenen Stadtbewohner ausgerichtet waren. Daraus leitet er Planungsempfehlungen $\mathrm{ab}$, die dazu beitragen, einen gerechten und nachhaltigen Wiederaufbau in und von Städten nach Katastrophen zu leisten.

Allen Leserinnen und Lesern wünschen wir eine anregende Lektüre. 